\title{
APEXeditor: A Spreadsheet-Based Tool for Editing APEX Model Input and Output Files
}

\author{
Javier M. Osorio Leyton \\ Texas A\&M AgriLife Research, Blackland Research and Extension Center, Temple, TX, USA \\ Email: josorio@tamu.edu
}

How to cite this paper: Osorio Leyton, J.M. (2019) APEXeditor: A Spreadsheet-Based Tool for Editing APEX Model Input and Output Files. Journal of Software Engineering and Applications, 12, 432-446. https://doi.org/10.4236/jsea.2019.1210027

Received: September 3, 2019

Accepted: October 28, 2019

Published: October 31, 2019

Copyright $\odot 2019$ by author(s) and Scientific Research Publishing Inc. This work is licensed under the Creative Commons Attribution International License (CC BY 4.0).

http://creativecommons.org/licenses/by/4.0/

(c) (i) Open Access

\begin{abstract}
The APEXeditor, an Excel-based tool has been developed using the Visual Basic for Applications (VBA) to provide a graphical user interface (GUI) to the Agricultural Policy Environmental eXtender (APEX) model. APEX, in its native form, requires users to edit text files for modifying input files; therefore a GUI interface can aid users in modification of these files and reduce errors. Microsoft Excel is a popular spreadsheet program that has the largest user base among scientists and researchers, thus providing a relatively common platform in which stages the tool. The APEXeditor requires minimal additional learning to operate the tool for those who already have basic level of knowledge in Excel. The user can load APEX input files into the spreadsheet tool and the GUI offers meta information and provides functions to edit, write, and run the APEX model. Ultimately, the APEXeditor substitutes existing GUI programs such as WinAPEX or ArcAPEX that require installation or additional licensing. A series of scripts were developed as a back-end engine that automates data formatting and editing of linked APEX input ASCII files including database libraries. The simple architecture of the tool helps users maintain the quality of the data and allows error-free editing of APEX model inputs to characterize the system under study. This tool is suitable for all kinds of applications and has been successfully used for the creation of APEX model runs in numerous studies.
\end{abstract}

\section{Keywords}

Simulation Models, APEX, Excel, Visual Basic, Graphical User Interface

\section{Overview}

Simulation models have long been used in agriculture with varying levels of success. These models are often designed to capture the most important features of a given agricultural system that adequately represent a complex system. Howev- 
er, the complexity of the system often leads to a greater complexity in the model structure, with a large input of data required to simulate such systems. Models that represent agricultural systems have become indispensable tools for designing management practices, evaluating sustainable production, and minimizing environmental stresses.

The Environmental Policy Integrated Climate Model (EPIC) [1] and the Agricultural Policy Environmental eXtender-APEX [2] are versatile and useful tools for evaluating complex landscape and management scenarios. EPIC is a field-scale model designed to simulate homogeneous areas in terms of weather, soil, topography, plant cover and management [3]. APEX is a multi-field landscape model that essentially simulates multiple spatially explicit distributed fields with EPIC [4] with interactions among the fields. APEX and EPIC are designed to estimate how climate and management practices influence soil-plant-water-nutrients relations. The models account for major physical and biological processes of agricultural systems, including hydrology, crop growth, and yield, water balance, nutrients transformations, storage, and transport (nitrogen, phosphorus and carbon), soil loss fate and transport, etc. [3]. Both models have been used under numerous conditions to assess agricultural systems across a broad range of landscapes at different spatial scales, design sustainable land management interventions, environmental impact studies, etc. [4] [5]. Because of APEX's solid background and capabilities, it is used to support modeling needs in projects funded by the U.S. Department of Agriculture (USDA) and the U.S. Department of the Interior (USDI) among others. For instance, APEX has been used since 2002 by the USDA's Natural Resources Conservation Service (NRCS) in the Conservation Effects Assessment Project (CEAP) for estimating the environmental benefits and effects of conservation practices on agricultural landscapes and developing the science base for managing the agricultural landscape for environmental quality across the United States [6]. Likewise, APEX is also used for regional quantification of land management in 54 million acres of public lands by the USDI's Bureau of Land Management (BLM) Colorado River Basins Salinity Control Program and to maintain and reduce salt in the Colorado River based to meet Salinity Act requirements [7].

APEX is built on an open-source FORTRAN programming language. It reads and writes input and output (I/O) in text files. The APEX text file formats are however not homogenous among the different types of input files. Formatting requirements for I/O files are complicated as described in the APEX user's manual [8]. Some I/O files are in fixed-format while others are in free-format or a combination of both, which is often a significant challenge to inexperienced model users. While all files can be created and edited with a text editor (e.g., Microsoft Notepad), the complexity of the file structure and the rigid formatting requirements make this approach rather difficult and prone to errors. This aspect of APEX can be cumbersome and frustrating during model execution, and it is the main reason why graphical user interfaces (GUI) are needed. However, GUIs for APEX have not been the main focus of the developers of EPIC and 
APEX.

Currently available Windows-based GUIs for APEX are ArcAPEX, WinAPEX, and i_APEX. ArcAPEX was developed as an ArcGIS extension that integrates GIS capabilities and algorithms with APEX databases [9]. The most current version of ArcAPEX conducts automatic delineation of stream network and sub-watersheds for large watersheds using ArcObjects and the Microsoft Visual Basic .NET. ArcAPEX must run within ESRI's ArcGIS 10.3.1 that requires licensing of main program and extensions. WinAPEX is a Windows-based desktop application developed by Texas A\&M AgriLife Research to manipulate model's inputs files for small watershed model applications [10]. Currently, WinAPEX is a deprecated version written in Visual Basic 6; however, developers are working to create a redesigned version of this interface using Microsoft Visual Basic .NET. Lastly, the interactive APEX (i_APEX) is another interface for the APEX model programmed in C++ [11]. The greatest advantage of i_APEX is its ability to perform multiple model runs. These GUI programs offer a "guided" workflow to create a watershed model and related input files for APEX simulation. These applications use Microsoft Access to store data in tables. With a model Run action button, the interfaces overwrite existing text files with a format that the models recognize when executed. While these interface programs are capable of writing input files, neither of them offers capacity to edit existing APEX watershed datasets in ASCII format.

The objective of this short note is to describe a simple, yet powerful user interface for the management of the APEX input data (read and write), execution of individual APEX runs, and loading output files into the spreadsheet for further processing and visualization. The tool provides a well-organized interface with definitions and help assistance for most variables. This tool is suitable for all kinds of applications and has been successfully used for the creation of APEX model runs in numerous studies.

\section{Design, Features and Tool Capabilities}

The overall purpose of the APEXeditor is to use Microsoft Excel's grid capabilities as a front end to allow the user to manage both the input and output data required for APEX simulations. This tool facilitates the creation and modification of the APEX model's text files (inputs and outputs).

\section{Software environment}

The APEXeditor is written in Visual Basic for Applications (VBA) and Microsoft Excel 2010 and newer. It is aimed to work with IBM compatible personal computers using operating system Microsoft Windows 7 or Windows 10. The execution of the tool's VBA scripts does not require any administrator access token to work. This application takes advantage of Excel's structure and power to design simple user interfaces. In addition, the tool lets the users visualize and manipulate selected model outputs. The code is open source to encourage collaborative development that will enhance the capabilities of the tool. The auto- 
mated capabilities to write properly formatted input files aims to guarantee the reproducibility and reliability of the APEX projects created with the tool. The APEXeditor is constructed in a workbook with 29 worksheets. Each worksheet is designated to handle a corresponding APEX input or output file type with VBA scripts (Figure 1). All actions are executed within the corresponding worksheet. The user can read and write APEX files, similar to the Microsoft Windows file management menu options for "Open" and "Save". The user can also execute the model from within the tool to test the modifications made. The tool is currently compatible with APEX1501, which is the open source version of the APEX made available to the scientific community.

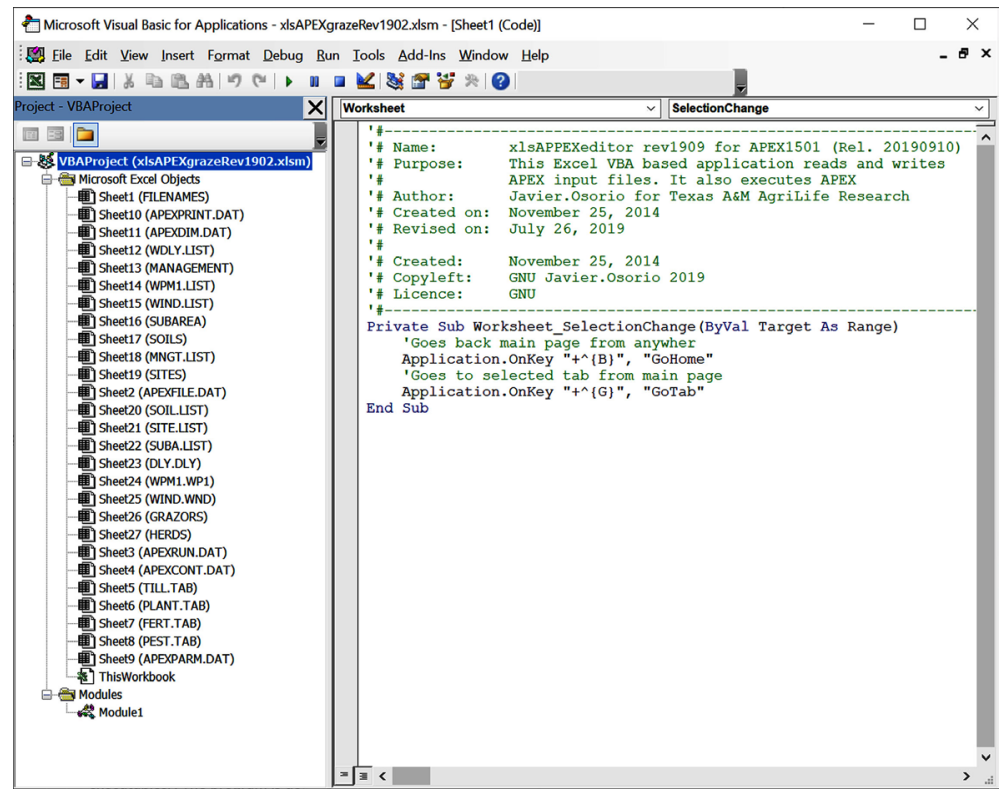

Figure 1. Organization of the VBA modules represented by APEXeditor.

\section{APEXeditor architecture}

The tool has the same structure as that of the APEX model. The conceptual architecture of the APEX file system is shown in Figure 2. APEX has four different kinds of files: tables, lists, data files, and output files. The model works as follows: the executable (APEX.exe) reads first the APEXDIM.DAT to set the dimensions of dynamic internal arrays; then, the model opens the APEXFILE which is a list of filenames that provide a reference for APEX in determining what data is to be used for each run (tables, lists and the parameters file). Then the model reads the controlling file, APEXCONT.DAT, which defines the user's selection of methods and constants that control the model runs defined in the APEXRUN.DAT. APEX gets the information that characterizes the run to be made from the APEXRUN.DAT and a combination of lists and data files. Lists are a repository of a combination of index numbers and data file names. The model uses a system based on pointers to access the information from each data file. For instance, from the subarea file the model points to the soils list (SOILLIST.LIS) with an index, this index is associated with a soils file name that 
is open to obtain all the information related to physical and chemical properties of the soil assigned to that specific part of the study area, etc.

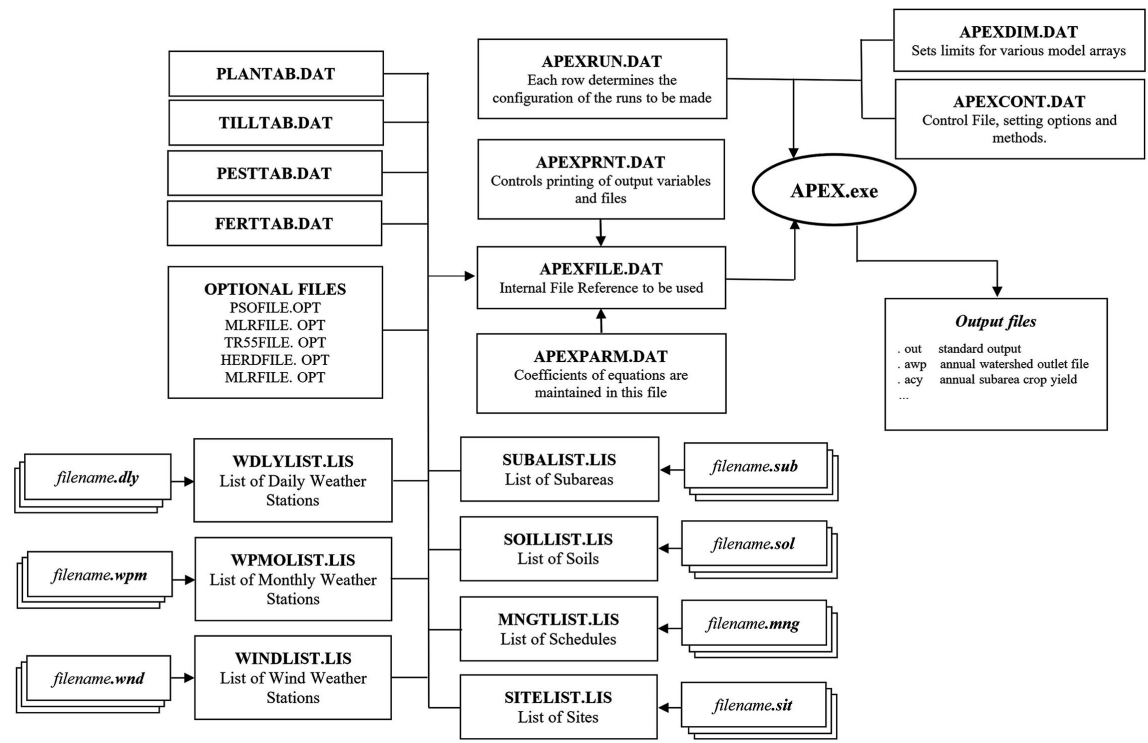

Figure 2. Architecture of the APEX file system (Modified from [8]).

The APEXeditor is structured in one independent Excel Workbook. The editing interface for each model input file is set in different worksheets within the Excel Workbook. The code reads and writes APEX text files that are written with different formatting characteristics. The following input files are written with a Fixed Width Format: APEXFILE, APEXPRNT, APEXPARM, SITEFILE, SOILFILE, MNGTFILE, WINDFILE, WPMOFILE, TILLTAB, PLANTAB, PESTTAB, and FERTTAB. On the other hand, files written as space-delimited (also called free format) are: APEXDIM, APEXRUN, APEXCONT, SUBAFILE, SOILLIST, MNGTLIST, WPMOLIST, WWINDLIST, SITELIST, SUBALIST, and WDLYLIST. Regardless of the formatting, both types of files have a data structure that is highly suited for Microsoft Excel. The tool takes advantage of the spreadsheet's grid to display the values in an organized manner.

A "Get Files" button within the editing interface uses the extension portion of the APEX file name to identify all similar files that will populate the drop-down menu and are available for editing. For example, in the case of the files with a "DAT" extension, the algorithm searches for a common pattern in the name of the file, for instance, if "* $\mathrm{RUN}$ " " were entered, the code will search for all the files that have the keyword "run" in the file name (i.e. APEXRUN.DAT) and displays these for the user in a drop-down menu. The user can then select which file to edit (i.e. read and write).

The APEXeditor code has two different algorithms for reading each kind of input file. Every file is read line by line until the end of the file. In the case of fixed-width format, the lines are parsed one at a time, taking into account the number of characters used in the model's code to assign values to each variable. 
Likewise, in the case of space-delimited files, the tool uses a double for-loop to read each line and then parse the line searching for non-white spaces. The algorithm for reading the fixed-width format files disregards one or more spaces in-between values, and because of that, the user should enter zeros instead of leaving the cells empty. The writing algorithm of the tool is common for all files, and it prints formatted input files in concordance with the specification of each file.

The software includes a quality tested reference data set (Figure 3), that fully match the file naming conventions used in this publication (Figure 4). The files can be used as a template to create a new APEX project or individual files. The user can opt not to use the provided set of files as reference data or define an alternative data set, which can be partly or entirely independent of the above dataset.

\begin{tabular}{|c|c|c|c|c|}
\hline \multicolumn{3}{|l|}{$\mathbb{I I}|\mathbb{E} \mathbb{\|}=|$ | |lites } & \multirow[t]{2}{*}{ 口 } & \multirow{2}{*}{$\begin{array}{l}x_{0} \\
v 0\end{array}$} \\
\hline File Home Shar & e view & & & \\
\hline$\leftarrow \rightarrow \vee \uparrow \| \propto A$ & APEXedito..., IOfiles & $\sim 0 \mathrm{se}_{\mathrm{s}}$ & Search Lofiles & $\circ$ \\
\hline Name & Date modified & Type ^ & Size & \\
\hline (1) APEX1501.exe & 8/6/2019 4:22 PM & Application & & 838 кв \\
\hline$\triangle A$ APEXCONT.DAT & 7/31/2019 4:11 PM & DAT File & & 1 кв \\
\hline APEXDIM.DAT & 7/31/2019 2:07 PM & DAT File & & $1 \mathrm{kB}$ \\
\hline$\triangle A$ APEXFILE.DAT & 8/5/2019 10:42 AM & DAT Flle & & $1 \mathrm{kB}$ \\
\hline APEXPARM.DAT & 7/31/2019 2:08 PM & DAT File & & $2 \mathrm{~KB}$ \\
\hline APEXPRNT.DAT & 7/31/2019 2:08 PM & DAT Flle & & 2 кв \\
\hline APEXRUN.DAT & 7/26/2019 4:54 PM & DAT File & & $1 \mathrm{kB}$ \\
\hline adyrile.dly & 7/30/2019 1:23 PM & DLYFile & & 286 Кв \\
\hline MNGTUST.LIS & 9/3/2019 10:12 AM & us File & & $1 \mathrm{kB}$ \\
\hline SITELIST.LIS & 7/31/2019 2:20 PM & US File & & $1 \mathrm{kB}$ \\
\hline D soluIST.LIS & 9//2019 10:00 AM & US file & & $1 \mathrm{kB}$ \\
\hline A) SUBALITI.LIS & 7/31/2019 4:18 PM & US File & & $1 \mathrm{kB}$ \\
\hline WDIVISTT.LIS & 9/3/2019 9:55 AM & us file & & $1 \mathrm{kB}$ \\
\hline ä WINDLIST.LIS & 9/3/2019 9:59 AM & us file & & $1 \mathrm{kB}$ \\
\hline DI WPMOLIST.LIS & 9/3/2019 9:59 AM & uS File & & $1 \mathrm{kв}$ \\
\hline al mngttilemng & 7/31/2019 2:10 PM & MNG File & & $3 \mathrm{kB}$ \\
\hline D HERDFILE.OPT & 11/22/2013 9.27 PM & OPT File & & $1 \mathrm{kB}$ \\
\hline DIRIRNFILEOPT & 6/5/2013 3:30 PM & OPT File & & $1 \mathrm{kB}$ \\
\hline DOSOFILEOPT & 6/5/2013 3:38 PM & OPT File & & о Кв \\
\hline D RFDTFLE.OPT & $11 / 25 / 2013455 \mathrm{PM}$ & OPT File & & 1 кв \\
\hline TRSFFILE.OPT & 6/5/2013 3:35 PM & OPT File & & $6 \mathrm{~KB}$ \\
\hline sitefles sit & 7/31/2019 2:13 PM & SIT file & & $1 \mathrm{kB}$ \\
\hline a soiffe sol & 7/31/2019 2:10 PM & SOL File & & $5 \mathrm{~KB}$ \\
\hline al subafilesub & 7/31/2019 6:13 PM & SUB File & & $2 \mathrm{~KB}$ \\
\hline$\triangle$ APEXEEFTTAB & 7/31/2019 2:14 PM & TAB File & & $7 \times \mathrm{KB}$ \\
\hline$\triangle A P E X P E S T$ TAB & 7/31/2019 2:15 PM & TAB Fle & & $38 \mathrm{kB}$ \\
\hline APEXPLITITAB & 7/30/2019 6:59 PM & TAB File & & $85 \mathrm{kB}$ \\
\hline A & 7/31/2019 2:16 PM & TAB File & & $160 \mathrm{~KB}$ \\
\hline an windfile.wnd & 7/31/2019 2:19 PM & WND File & & 2 кв \\
\hline whrfilewp 1 & 8/2/2019 12:17 PM & WP1 File & & $2 \mathrm{kB}$ \\
\hline 30 items & & & & 狦 \\
\hline
\end{tabular}

Figure 3. Example of files included in a simple APEX project.

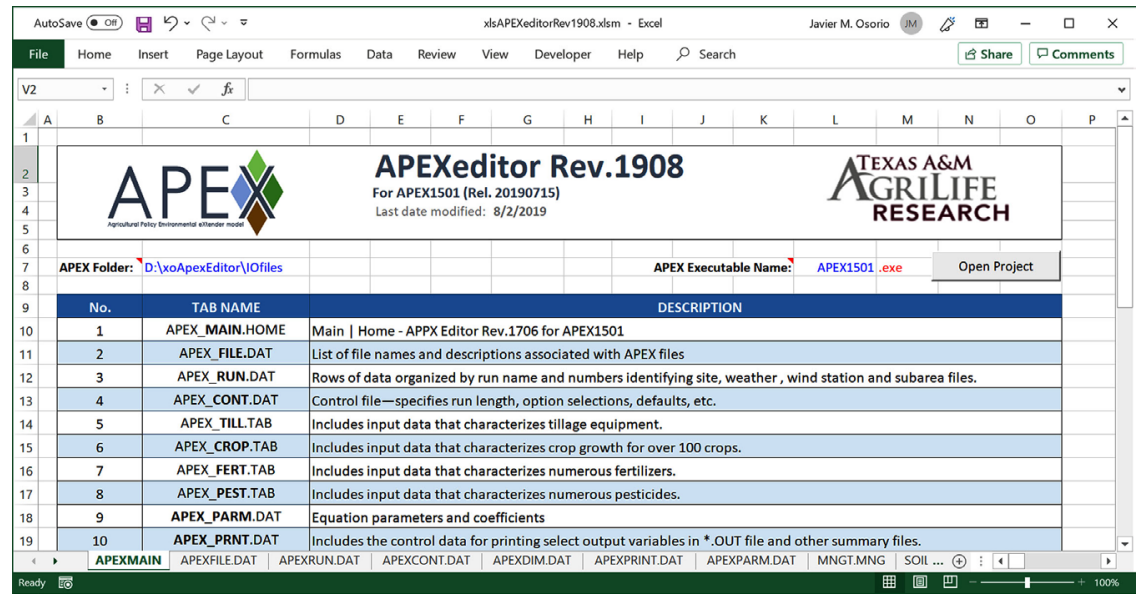

Figure 4. Main window that allows users to set the path to the APEX project, set the name of the model's executable and open an APEX project. 


\section{Details of the main features}

The main window (APEXMAIN) presents a list of all files that can be accessed with this interface. In this window, the user has the option to define the path to the APEX project folder (the folder in which the input files and the APEX executable are located) and the name of the model's executable file (default is APEX.exe). Each worksheet can be edited independently, as long as the APEX file and the four tables required as a minimum by the model are loaded (plant, fertilizers, management, and pesticides). This is because some file's names that the tool needs are obtained from the APEXFILE worksheet and also, the management worksheet uses the tables as a cross-reference for identification of management actions and to display specifics about them. For a fast load of a project, there is an action button named "Open Project" that will open all the control files, tables, and lists within the APEX project folder. Finally, the user can navigate to each of the worksheets by selecting the name of the worksheet and the hotkey combination CTRL + G (control + go to). Likewise, the user can return to the main worksheet from any worksheet by the key combination CTRL $+\mathrm{B}$ (control + back).

Figure 5 shows the features and objects that are common to all worksheets. The "Read" and "Write" action buttons can be found in all worksheets. A "Run" button will execute APEX.exe from all worksheets that lets the user update inputs, such as data files and tables, and then execute these to evaluate outputs. For all the files that are not unique to the model (e.g. soils, management, etc.), the interface has an "Get Files" action button that identifies and retrieves all files that share the same file extension or same name’s root (e.g., ${ }^{\star}$ PARM ${ }^{\star}$.DAT) and populates all instances in the drop-down list menu from which the user can select the file to be edited. The action button "Read" reads the selected file and loads the input variables into the spreadsheet that contains the variable name, a short description of the variable, and a suggested range of values for the variable (Figure 5). This makes it easy for users to modify input variables with meta information and guidance on suggested ranges. Otherwise, the user would have to deal with using the APEX manual or other documents that identify variable names, their positions, formatting requirement, and the upper and lower limits defined for the variable. Some of the variables' name will have an Excel's notes feature denoted by a red triangle flag in the top right corner of the cell. Clicking on the cell automatically display the comment's content with additional information for a given variable, for instance, the message of the IET will describe all the different methods available in the APEX model to estimate potential evapotranspiration (PET). The numeric values for each variable can be modified in each cell by the user. The user can then click on the "Write" action button and the APEXeditor will write the changes into the respective APEX parameter file with the proper formatting. This action is equivalent to "Save" in other Microsoft Windows applications (i.e. NotePad, Word, etc.). To save the information with a different file name other than the default name (i.e., "Save As"), the user should enter a new filename next to the "New File Name" option, underneath 
the drop-down object within the worksheet. Two actions will occur: 1) a new file will be created with the name as entered in the cell and 2) the new file's name will be added to the drop-down menu.

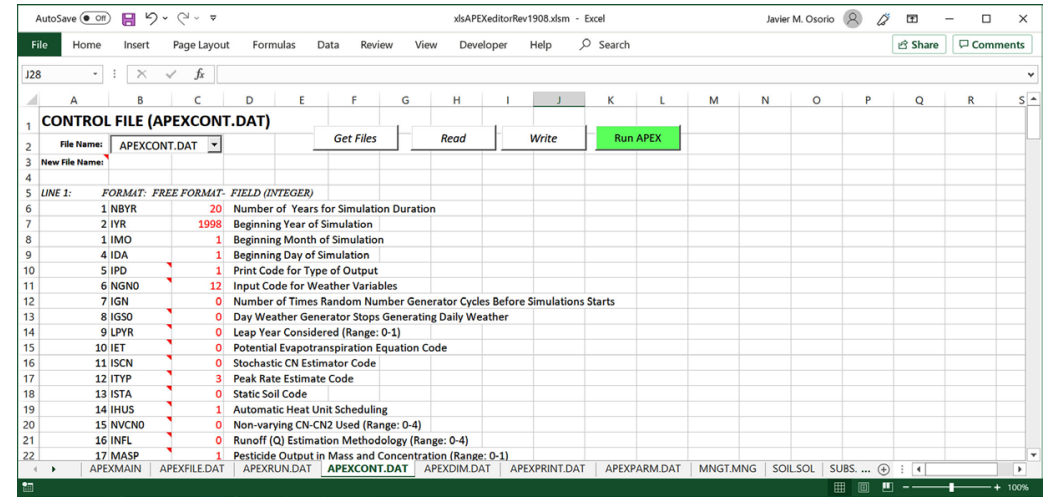

Figure 5. Representation of the most common objects and interface features of the APEXeditor.

In addition to the editing capabilities, the APEXeditor keeps the functionality of Excel for plotting and data manipulation, such as sort, conditional formatting, copy/paste/insert, filtering, etc. Filtering can be useful in the case of working with APEX's tables (Figure 6). For instance, the tables can be filtered, to narrow down the options of equipment for harvesting, or selecting only annual crops, etc. Figure 6 shows the features and table like disposition that are common to all four of APEX's main tables (APEXTILL.TAB, APEXPLANT.TAB, APEXFERT.TAB and APEXPEST.TAB). The operations table (APEXTILL.TAB) provides parameters that detail field management activities and related equipment commonly used in agricultural operations (28 parameters per line). The APEXPLANT.TAB table stores parameters that characterize the plant growth for different plant species (Figure 6). Each line in this table represents a plant species characterized by 56 plant growth parameters. Likewise, nine parameters that characterize the most common fertilizers used in agricultural management (inorganic and organic) are listed in the APEXFERT.TAB. Finally, the pesticide table, APEXPEST.TAB, includes the most common pesticides used in agricultural management, with each pesticide having six parameters per line.

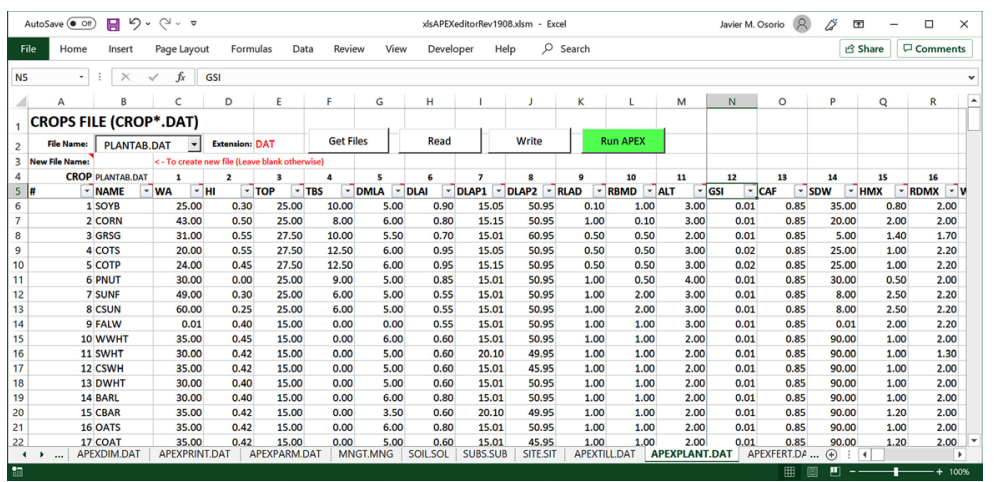

Figure 6. Example of a table-like file (APEXPLANT.TAB) with the filter feature enabled. 
Without using the APEXeditor, one of the most problematic files to work with is the daily weather file (Figure 7). The APEX model requires dates (year, month and day) and weather data such as solar radiation $\left(\mathrm{MJ} / \mathrm{m}^{2}\right)$, maximum and minimum temperature $\left({ }^{\circ} \mathrm{C}\right)$, precipitation $(\mathrm{mm})$, relative humidity (fraction), and wind speed $(\mathrm{m} / \mathrm{s})$. The daily weather file has restrictions due to the fixed-width format of the file with the columns related to weather parameters being six characters wide. This means than only four or five characters are available for the numeric portion of the data because one space is taken by the decimal point and one space can be taken for the negative symbol (e.g., negative temperatures). Whenever this formatting condition is not met, the model crashes with an error message that points to the daily weather file. In addition, APEX identifies missing values in two different ways depending on the weather parameter. Missing values for temperature and precipitation are denoted by a 999 series, while missing values for solar radiation, relative humidity, and wind velocity must be set to blank. This means that if 999 is erroneously set in the solar radiation column, the model will use $999 \mathrm{MJ} / \mathrm{m}^{2}$ in that given day.

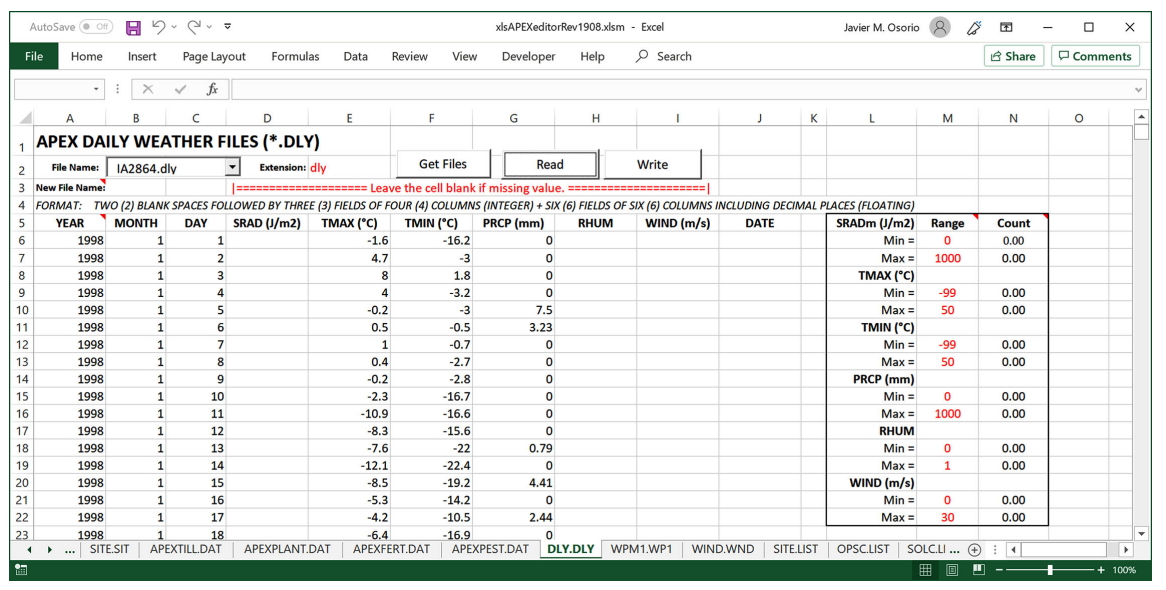

Figure 7. Worksheet that allows users to add or modify historical daily weather data.

Figure 7 depicts the APEXeditor worksheet where users can read, edit and create daily weather files for APEX. The Write action button will save the data in the correct format. Positive decimal values with more than 5 digits and negative values with more than four digits are truncated accordingly. The user should leave blank cells for missing values (regardless of the parameter), the tool will write the missing values correctly for each parameter. Also, the APEXeditor will generate a worksheet that that displays erroneous values in the time series. This is not meant to be used to identify outliers, but is intended to display values that do not exist in nature (e.g. temperatures greater than $50^{\circ} \mathrm{C}$ ). The tool displays maximum and minimum values for all six parameters. In addition, the APEXeditor will count the total number of values in the time series that are outside a user-defined range. The user can use this count as a warning to use the sorting and filtering capabilities of Excel to correct the erroneous values. If no action is taken, the Write button will write the daily weather file replacing the values with 
the ranges set in this worksheet.

The calendar sequence of field activities in each subarea or field are described with the management operation schedule file (Figure 8). The model can perform a total of 29 different management actions (i.e. plowing, sowing, planting, harvesting, grazing, etc.). For each action, where each line represents an action that happens on a given date, the APEX model uses a series of indexes and pointers to associate the information provided from the different tables. Also, each action can be further described using the context specific operation parameters $\left(\mathrm{OPV}_{0}\right.$ to $\left.\mathrm{OPV}_{7}\right)$; therefore, each action uses the parameters differently. For instance, a fertilizer application will need the fertilizer to be used from the fertilizers table $\left(\mathrm{OPV}_{0}\right)$ and the application rate (expressed in kilograms per hectare) will be set using $\mathrm{OPV}_{1}$. Whereas, for grazing a start or stop grazing date need to be entered, in this case $\mathrm{OPV}_{0}$ is the herd identification code in the list of available herds, and $\mathrm{OPV}_{1}$ is the stocking rate expressed in area per animal head (ha/head).

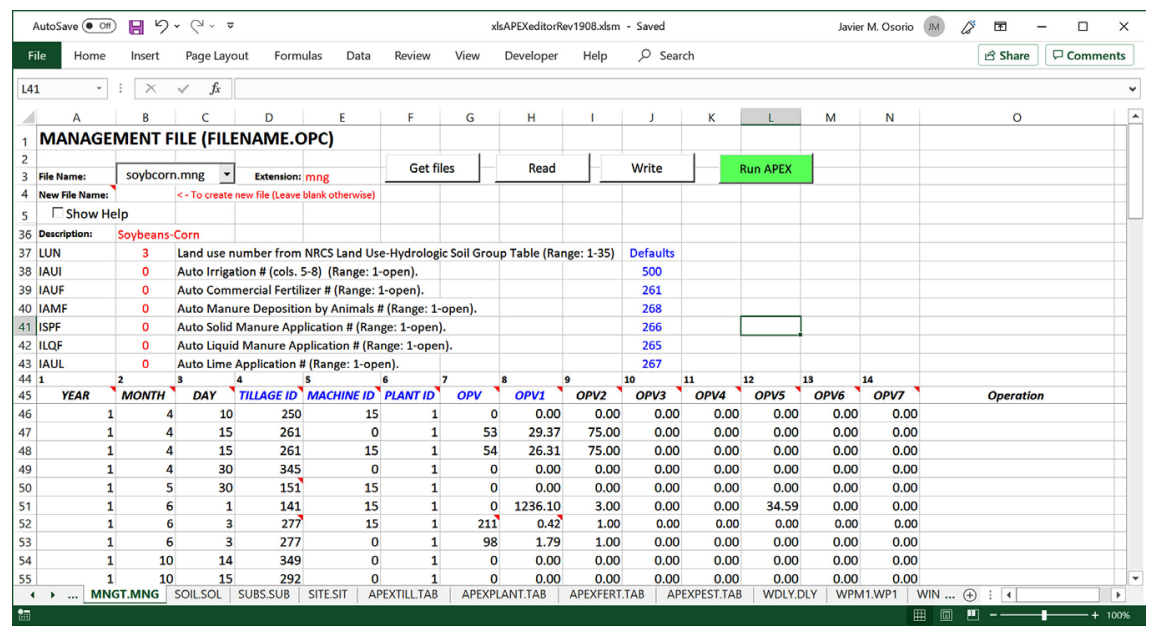

Figure 8. Interface that allows users to edit/modify the APEX management file.

A series of red triangle flags, denoting Excel's note boxes, are used to describe each of the parameters in the management operations file. The spreadsheet also includes a checkbox to display additional information to assist in building or modifying the management files. When the checkbox is selected, a table with all 29 possible management actions is displayed. Each line in the table represents a given action and the default values for each required parameter that characterize each action (Figure 9). The "Tillage ID" column values in the management table are color-coded in red and blue. The values in red indicate if there is more than one type of equipment used to perform that given action. Therefore, the user has to select the appropriate one from the management table. Conversely, the values coded in blue indicate that the action has no additional options and hence there is no need to edit the set column values. Likewise, the OPV parameters are also color-coded in gray and solid black. Solid black values mean that a given action requires a value to be entered in that column. For each action, the OPV parame- 
ters that are not in use are grayed out. In addition, each OPV-parameter in use also has a triangular red flag which denoting Excel's note boxes that will display a short pop-up message that describes the role that the OPV plays in that given action. The user can copy the entire line and paste it into the schedule. The user can then proceed to modify the dates and values for the required parameters.

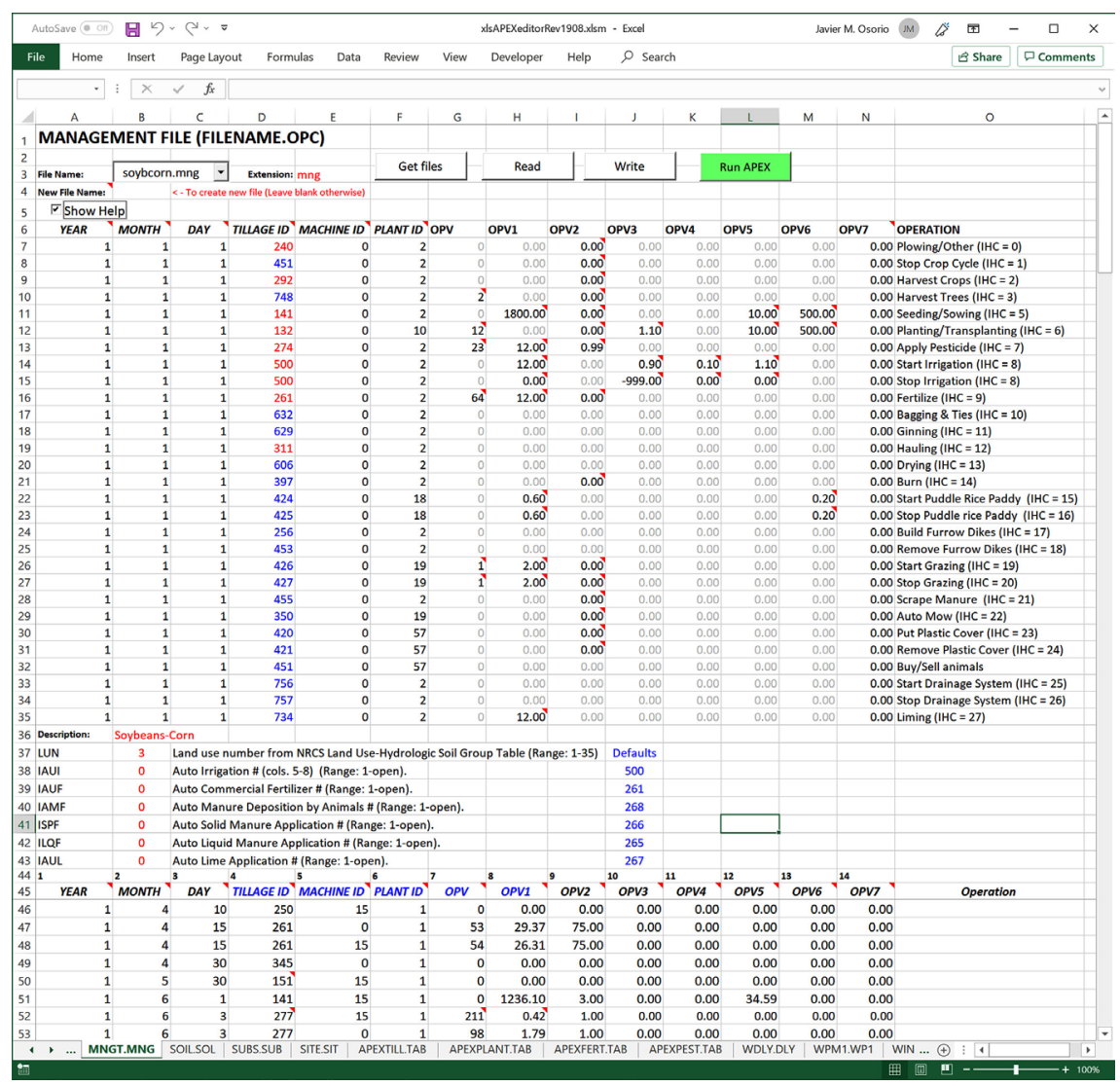

Figure 9. Management file worksheet depicting the help feature enabled. The "Show Help" feature allows users to view the different field operations that APEX is able to simulate and copy these to their management operations schedule.

Other APEX input files that can also be edited with the APEXeditor include: APEXDIM.DAT, APEXRUN.DAT, APEXCONT.DAT and SITEFILET.DAT. APEXDIM.DAT is a collection of numeric variables for setting the dimension of various arrays that the internal APEX code uses to store variables. The APEXRUN.DAT file defines the configuration of the runs to be made. Through this worksheet in the APEXeditor, the user can define the run names (a variable that is used to name the output files) and numbers from list files that specify the site, weather parameter, and subarea files etc. The APEXCONT.DAT file contains a selection of methods and constants that are pertinent to the entire simulation period and are common to all subareas and sites. While the SITEFILET.DAT file is relevant in APEX, most of the variables in this file can be set from other files (i.e. APEXCONT.DAT), therefore most users will not need to edit this file in the APEXeditor. 
An important APEX input file is the subarea file ("subafile.sub"). In APEX, each subarea or Hydrologic Landuse Unit (HLU) is homogenous with regard to climate, soil, land cover, and topography. The information related to each HLU can be characterized in the file subafile.sub using the APEXeditor. From this file, the user can point to the soils data, daily weather data, and the operation schedule to be used in each HLU. A subarea may be linked with several other subareas according to the water routing direction in the watershed, starting from the most distant subarea and moving towards the watershed outlet. The user can also edit existing monthly weather stations that describe weather-related statistics for the subareas.

The APEXPARM.DAT file contains many coefficients of equations used in APEX. The APEXPARM.DAT file is available to be edited in the APEXeditor. However, users should use caution when editing this particular file due because APEX is sensitive to slight changes in the parameters contained in this file. Information about the parameters is available in the form of Excel's note boxes and includes the equation used in the APEX code in which the parameter is used. The ability to examine the equation used assists in defining and explaining the magnitude of change that would result from modifying the coefficient parameters. The list files, such as the SUBALIST.LIS and SOILLIST.LIS are repositories or listings of specific files which can be referenced by an index number and associated file name (name and extension, e.g. soilsfile.sol). Figure 10 shows the template used to edit the following repositories with this tool: subareas, soils, management, sites, and weather (daily and monthly).

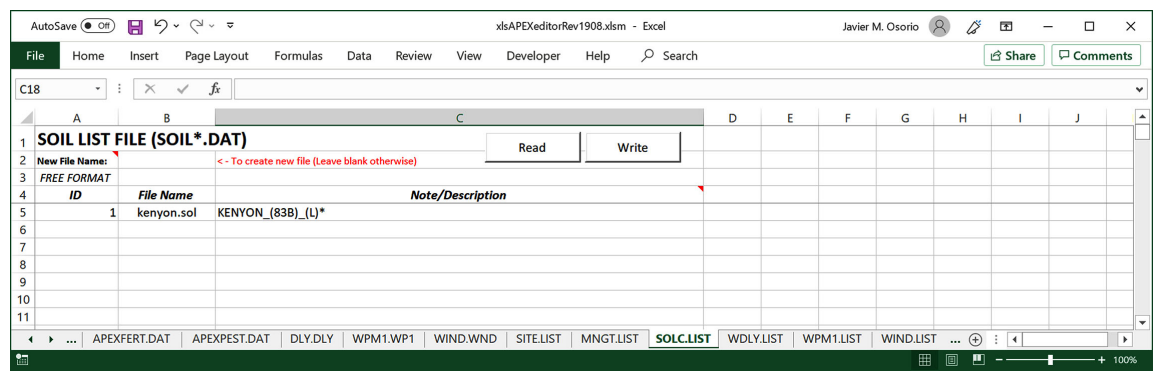

Figure 10. Example of a worksheet to edit list files.

When all of the input files have been properly set up, APEX.exe can be executed directly from the tool. Users can click on the "Run" button which will call the APEX executable specified in the main worksheet. If the cell is blank, the tool will attempt to execute the file APEX.exe. While the model can generate 50 different output files, the APEXeditor workbooks include two additional tabs for data evaluation, plotting and statistical analysis of two of the most frequently used model outputs: The Annual Water Balance (AWP) and the Annual Crop Yield (ACY) (Figure 11). The file APEXPRNT.DAT worksheet aims to facilitate retrieval, manipulation, and printing of APEX output files; it helps the user to select which output files will be generated by the APEX model and in some cases the variables to be added to the output files. 


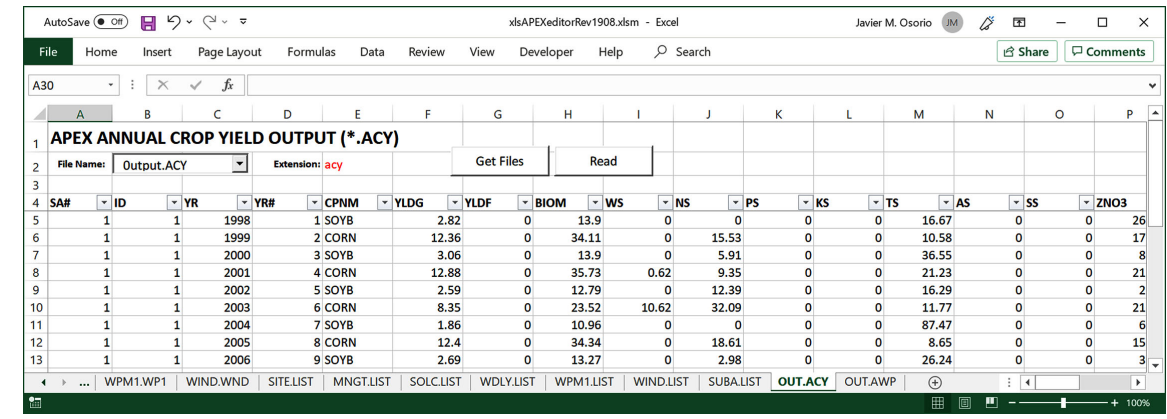

Figure 11. Representation of the annual crop yield output.

\section{Discussion and Conclusions}

The use of the application developed in Excel and written in VBA has been described and illustrated. The APEXeditor tool facilitates the work and creation of otherwise cumbersome APEX input files. Microsoft Excel is one of the most common and widely used spreadsheet applications. Scientists and researcher are familiar with Excel, so using the APEXeditor will not require additional learning to operate the tool. Simplification of writing input files for the APEX model allows the users to focus on the quality of the data to characterize the system under study, as well as, the analysis, evaluation and interpretation of the model results. Additionally, the flexibility of the tool reduces the steepness of the learning curve, which is expected to promote the use of a powerful simulation model, such as APEX. The software is built upon Microsoft Excel; therefore, its realization depends on the Microsoft Office platform. Since the tool's initial development in 2014, it has been used extensively for research work and in APEX training workshops throughout the world, which has resulted in numerous directly associated scientific publications [12]-[23].

\section{Availability and Feedback}

APEXeditor is available free of charge for non-commercial uses. The application and associated model executables can be downloaded from

http://epicapex.tamu.edu/model-executables/. The program is distributed along with a Getting Started manual and a reference dataset that can be used as a template to build new projects. Comments and suggestions about APEXeditor may be sent to josorio@tamu.edu.

\section{Acknowledgements}

During the development of this tool, Drs. Luca Doro, Manyowa Meki and Jaehak Jeong provided useful insight into the design of the interface and invaluable proof testing of the tool. In addition to testing the tool Drs. Jay Angerer, Manyowa Meki and Jaehak Jeong have also contributed to the writings and proof reading of this document. All are affiliated with Texas A\&M University-AgriLife Research, Blackland and Research Center at Temple, TX 76502, USA. This development did not receive any specific grant from funding agencies 
in the public, commercial, or not-for-profit sectors. Funding for preparation of this manuscript was provided by Dr. Jay Angerer under USDA National Institute of Food and Agriculture, Hatch/Evans-Allen/McIntire Stennis project 1009337.

\section{Conflicts of Interest}

The author declares no conflicts of interest regarding the publication of this paper.

\section{References}

[1] Williams, J.R. (1990) The Erosion Productivity Impact Calculator (EPIC) Model: A Case History. Philosophical Transactions of the Royal Society of London B, 329, 421-428. https://doi.org/10.1098/rstb.1990.0184

[2] Williams, J.R. and Izaurralde, R.C. (2005) The APEX Model. BRC Rep. 2005-02. Blackland Res. Center, Texas A\&M Univ., Temple. https://doi.org/10.1201/9781420037432.ch18

[3] Wang, X., Williams, J.R., Gassman, P.W., Baffaut, C., Izaurralde, R.C., Jeong, J. and Kiniry, J.R. (2012) EPIC and APEX: Model Use, Calibration, and Validation. Transactions of the American Society of Agricultural and Biological Engineers, 55, 1447-1462. https://doi.org/10.13031/2013.42253

[4] Taylor, R.A.J., Jeong, J., White, M. and Arnold, J.G. (2015) Code Modernization and Modularization of APEX and SWAT Watershed Simulation Models. International Journal of Agricultural and Biological Engineering, 8, 81-94.

[5] Gassman, P.W., Williams, J.R., Wang, X., Saleh, A., Osei, E. and Hauck, L.M. (2010) Invited Review Article: The Agricultural Policy/Environmental eXtender (APEX) Model: An Emerging Tool for Landscape and Watershed Environmental Analyses. Transactions of the American Society of Agricultural and Biological Engineers, 53, 711-740. https://doi.org/10.13031/2013.30078

[6] U.S. Department of Agriculture, Natural Resources Conservation Service (2017) Effects of Conservation Practices on Water Erosion and Loss of Sediment at the Edge of the Field: A National Assessment Based on the 2003-06 CEAP Survey and APEX Modeling Databases. $74 \mathrm{p}$.

[7] Boyd, R. and Green, C. (2018) A Framework for Improving the Effectiveness of the Colorado River Basin Salinity Control Program 2018-2023. Bureau of Land Management National Operations Center, Denver, 20 p.

[8] Steglich, E.M., Osorio, J., Doro, L., Jeong, J. and Williams, J.W. (2018) Agricultural Policy/Environmental eXtender Model User Manual Version 1501. Blackland Research and Extension Center, Temple. https://epicapex.tamu.edu/manuals-and-publications/7

[9] Tuppad, P., Winchell, M.F., Wang, X., Srinivasan, R. and Williams, J.R. (2009) ArcAPEX: ArcGIS Interface for Agricultural Policy Environmental Extender (Apex) Hydrology/Water Quality Model. International Agricultural Engineering Journal, 18, 59-71.

[10] Magre, M., Williams, J.R., Harman, W.L., Gerik, T., Greiner, J., Francis, L., Steglich, E. and Meinardus, A. (2006) WinAPEX Users Guide Version 1.0. Blackland Research and Extension Center, Texas A\& M System, Temple. http://agrilife.org/epicapex/files/2014/09/winapexv0604_nov2006.pdf

[11] Siemers, M., Plotkin, S. and Gassman, P.W. (2014) Interactive APEX (i_APEX) Us- 
er's Guide Using APEX2110 and APEX0806. CARD Technical Reports, 40.

[12] Clarke, N., Bizimana, J.C., Dile, Y., Worqlul, A., Osorio, J., Herbst, B., Richardson, J.W., Srinivasan, R., Gerik, T.J., Williams, J., Jones, C.A. and Jeong, J. (2016) Evaluation of New Farming Technologies in Ethiopia Using the Integrated Decision Support System (IDSS). Agricultural Water Management, 180, 267-279. https://doi.org/10.1016/j.agwat.2016.07.023

[13] Doro, L., Jones, C., Williams, J.R., Norfleet, M.L., Izaurralde, R.C., Wang, X. and Jeong, J. (2017) The Variable Saturation Hydraulic Conductivity Method for Improving Soil Water Content Simulation in EPIC and APEX Models. Vadose Zone Journal, 16, 1-14. https://doi.org/10.2136/vzj2017.06.0125

[14] Zilverberg, C.J., Williams, J.W., Jones, C., Harmoney, K., Angerer, J., Metz, L.J. and Fox, W. (2017) Ecological Modelling. Process-Based Simulation of Prairie Growth. Ecological Modelling, 351, 24-35. https://doi.org/10.1016/j.ecolmodel.2017.02.004

[15] Zilverberg, C.J., Angerer, J., Williams, J.W., Metz, L.J. and Harmoneye, K. (2018) Sensitivity of Diet Choices and Environmental Outcomes to a Selective Grazing Algorithm. Ecological Modelling, 390, 10-22. https://doi.org/10.1016/j.ecolmodel.2018.10.007

[16] Chavez, C.J., Enciso, J., Meki, M.N., Jeong, J. and Singh, V.P. (2018) Simulation of Energy Sorghum under Limited Irrigation Levels Using the EPIC Model. Transactions of the American Society of Agricultural and Biological Engineers, 61, 121-131. https://doi.org/10.13031/trans.12470

[17] Le, K.N., Jha, M.K., Reyes, M.R., Jeong, J., Doro, L., Gassman, P.W., Hok, L., de Moraes Sá, J.C. and Boulakia, S. (2018) Evaluating Carbon Sequestration for Conservation Agriculture and Tillage Systems in Cambodia Using the EPIC Model. Agriculture, Ecosystems \& Environment, 251, 37-47. https://doi.org/10.1016/j.agee.2017.09.009

[18] Worqlul, A.W., Dile, Y.T., Bizimana, J.C., Jeong, J., Gerik, T.J., Srinivasan, R., Richardson, J.W. and Clarke, N. (2018) Multi-Dimensional Evaluation of Simulated Small-Scale Irrigation Intervention: A Case Study in Dimbasinia Watershed, Ghana. Sustainability, 10, 1531. https://doi.org/10.3390/su10051531

[19] Zhang, L., Juenger, T.E., Osorio, J.M. and Behrman, K.D. (2018) Sensitivity Analysis of the APEX Model for Assessing Sustainability of Switchgrass Grown for Biofuel Production in Central Texas. BioEnergy Research, 11, 69-85. https://doi.org/10.1007/s12155-017-9878-8

[20] Meki, M.N., Kiniry, J.R., Worqlul, A., Kim, S., Williams, A.S., Reilley, J. and Osorio, J.M. (2019) A Field and Simulation-Based Assessment of Vetivergrass Bioenergy Feedstock Production Potential in Texas. (Manuscript Submitted for Publication)

[21] Sharifi, A., Lee, S., McCarty, G.W., Lang, M.W., Jeong, J., Sadeghi, A.M. and Rabenhorst, M.C. (2019) Enhancement of Agricultural Policy/Environment eXtender Model (APEX) Model to Assess Effectiveness of Wetland Water Quality Functions. Water, 3, 606-618. https://doi.org/10.3390/w11030606

[22] Zhao, J., Chu, Q., Shang, M., Meki, M.N., Norelli, N., Jiang, Y., Yang, Y., Zang, H., Zeng, Z. and Jeong, J. (2019) APEX Simulation of Spring Peanut Management in the North China Plain. Agronomy, 9, 443. https://doi.org/10.3390/agronomy 9080443

[23] Goehring, N. (2017) Modeling Salt Movement and Halophytic Crop Growth on Marginal Lands with the APEX Model (Master's Thesis). University of Nevada, Reno, NV.

https://scholarworks.unr.edu/bitstream/handle/11714/2761/Goehring_unr_0139M_ 12429.pdf? sequence $=1$ \&isAllowed $=\mathrm{y}$ 